\title{
Editorial
}

Gerald V. Goresky MD FRCPC, J. Roger Maltby MB FFARCS FRCPC

\section{Fasting guidelines for elective surgical patients}

is of interest to note that the addition of a small amount of cream or sugar to coffee or tea does not appear to cause significant delay in gastric emptying. ${ }^{8}$

There is particular interest in fasting guidelines for children because they tolerate a prolonged fast less well than adults. They do not understand rules which make them thirsty and hungry, and they may bypass nursing staff or parents to drink from the water fountain or to take another child's breakfast. The liberalization of guidelines for preoperative fluid administration offers the benefit of improved patient comfort ${ }^{6}$ and should result in fewer children demonstrating signs of dehydration at the time of induction of anaesthesia. Drinks containing milk should only be permitted for infants, for whom the timing of the last milk feed should be based on the child's age and feeding interval.

Will the alteration of guidelines for preoperative fluid administration alter the "risk" of anaesthesia? The purpose of the preoperative fast is to allow sufficient time for gastric emptying of ingested food and liquid, as aspiration of gastric contents into the lungs may cause aspiration pneumonitis. Gastric contents can only reach the pharynx if the lower oesophageal sphincter is incompetent or if vomiting occurs. Animal studies ${ }^{11,12}$ indicate that 0.4 $0.8 \mathrm{ml} \cdot \mathrm{kg}^{-1}$ of low $\mathrm{pH}$ must enter the lungs to cause fatal acid aspiration syndrome, although the volume of fluid present in the stomach for $0.4-0.8 \mathrm{ml} \cdot \mathrm{kg}^{-1}$ to enter the lungs is unknown. Ingestion of clear fluid does not affect the integrity of the lower oesophageal sphincter or the protective airway reflexes. Clear fluids could therefore only increase the risk of pulmonary damage if they increased residual gastric fluid volume or acidity; all studies demonstrate that this is not the case.

The volume and acidity of gastric contents are consistently reduced by histamine $\mathrm{H} 2$ receptor antagonists (cimetidine, ranitidine, famotidine), although there is no evidence that their routine use reduces the very low incidence of pulmonary aspiration in healthy elective patients. The advantage of using such agents is obvious for those patients who are at increased risk of aspirating gastric contents.
Department of Anaesthesia, University of Calgary, Calgary, Alberta. 
The Canadian Anaesthetists' Society, which previously recommended a five-hour fast for elective surgical patients, now recommends that each Department of Anaesthesia should formulate its own policies regarding preoperative fasting. ${ }^{13}$ Based on present knowledge, we suggest the following principles be applied to healthy patients scheduled for elective surgery:

1 No solid food should be ingested on the day of surgery.

2 Unrestricted clear liquids should be permitted until three hours before the scheduled time of surgery, and oral medications should be taken with $30 \mathrm{ml}$ of water up to one hour before surgery.

3 The preoperative administration of an H2-receptor blocker should be considered for patients who may be at increased risk of regurgitation and aspiration of gastric contents.

\section{Directives concernant le jeûne des patients lors d'une chirurgie élective}

La physiologie de base de la digestion et de la vidange gastrique a été décrite avant la découverte de l'anesthésie générale. Beaumont, en 1833, avait fait des observations de visu chez un homme ayant développé une fistule gastrique après une blessure par balle de l'estomac. ${ }^{1}$ Il a enregistré qu'un période pouvant atteindre cinq heures est requise pour que la nourriture solide soit convertie en chyme semiliquide et que celle-ci traverse le pylore; les liquide clairs se vidaient " aussitôt après leur ingestion ». En 1858, Snow a suivi les principes physiologiques en recommandant que l'opération soit faite «par le temps que le patient serait prêt pour un autre repas $" ; 2$ par la suite d'autres auteurs ont recommandé que les liquides clairs peuvent être ingérés deux à trois heures ${ }^{3}$ avant la chirurgie. ${ }^{4}$ La prescription de routine «à jeun après minuit" ignore tant la différence entre la vitesse de vidange gastrique entre les aliments solides et liquides clairs que les différences dans le temps de la cédule chirurgicale.

Si uniquement les liquides clairs sont consommés le jour de la chirurgie, ${ }^{5-8}$ les études cliniques récentes nous confirment constamment que le $\mathrm{pH}$ du liquide gastrique et son volume sont indépendants de la durée du jeûne liquide au-delà de deux heures. Au-delà de deux heures de l'ingestion de liquide clair, la sécrétion gastrique endo- gène est le principal déterminant $\mathrm{du} \mathrm{pH}$ du volume du contenu gastrique. Une plus grande durée de jeûne liquide n'améliore pas le milieu gastrique. Cependant le temps de l'administration des liquides préopératoires doit tenir compte des annulations ou des retards de la chirurgie.

En l'absence de facteurs pathologiques, l'ingestion de la plupart des liquides augmente la vitesse de vidange gastrique. ${ }^{9}$ A cause du temps variable et prolongé requis pour la vidange gastrique des solides, des corps gras et des liquides à pression osmotique élevée, ${ }^{10} \mathrm{il}$ apparaît prudent qu'ils ne soient pas consommés le jour de la chirurgie. Concernant les liquides clairs, ceux étudiés sont l'eau, les boissons gazeuses, les jus de fruits clairs, le thé et le café. Il est intéressant de noter que l'addition d'une petite quantité de crème ou de sucre au café ou au thé ne retarde pas significativement la vidange gastrique. ${ }^{8}$

Particulièrement intéressantes sont les directives de jeûne pour les enfants qui tolèrent moins que les adultes le jeûne prolongé. Ils ne comprennent pas les règles de la soif et de la faim et peuvent passer outre la surveillance du personnel infirmier ou des parents pour boire de l'eau de fontaine ou prendre le petit déjeûner d'un autre enfant. La libéralisation des directives pour l'administration préopératoire des liquides offre le bénéfice d'améliorer le confort du patient ${ }^{6}$ et doit diminuer le nombre d'enfants démontrant des signes de déshydratation lors de l'induction de l'anesthésie. Les liquides contenant du lait doivent être permis uniquement chez les nourrissons chez qui le temps de l'administration du lait doit être basé sur l'âge et l'intervalle des repas.

La reconsidération des directives concernant l'administration des liquides préopératoires amènera-t-elle un changement dans le « risque » de l'anesthésie? Le but du jeûne préopératoire est de permettre un temps de vidange gastrique suffisant pour éviter l'aspiration du contenu gastrique dans les poumons et la pneumonie d'aspiration. Le contenu gastrique peut atteindre le pharynx uniquement si le sphincter œsophagien inférieur est incompétent ou si le patient vomit. Des études animales ${ }^{11,12}$ indiquent que $0,4-0,8 \mathrm{ml} \cdot \mathrm{kg}^{-1}$ d'un $\mathrm{pH}$ bas doit entrer dans les poumons afin de déclencher un syndrome fatal d'aspiration acide et ce même si le volume de liquide actuellement présent dans l'estomac est alors inconnu. L'ingestion de liquide clair n'affecte pas l'intégrité du sphincter œsophagien inférieur ou les réflexes de protection des voies aériennes. Les liquides clairs peuvent ainsi uniquement augmenter le risque de dommage pulmonaire s'ils augmentent le volume ou l'acidité du liquide gastrique résidue ; or toutes les études démontrent que ceci n'est pas le cas.

Le volume et l'acidité du contenu gastrique sont réduits par les antagonistes des récepteurs $\mathbf{H} 2$ d'histamine (cimétidine, ranitidine, famotidine), même s'il n'y a aucune 
évidence que leur utilisation routinière réduit l'incidence déjà très basse d'aspiration pulmonaire chez les patients en bonne santé se présentant pour chirurgie élective. L'avantage de l'utilisation de ces agents est évident cher pour les patients présentant un risque accrue d'aspiration du contenu gastrique.

La Société Canadienne des Anesthésistes, qui précédemment recommandait un jeûne de cinq heures pour les patients devant subir une chirurgie élective, recommande maintenant la formulation d'une politique individuelle concernant le jeûne préopératoire par chaque département d'anesthésie. ${ }^{13}$ Se basant sur ceci, on suggère que les principes suivants soient appliqués aux patients en bonne santé cédulés pour la chirurgie élective :

1 Aucune nourriture solide ne doit être prise le jour de la chirurgie.

2 Des liquides clairs seraient permis jusqu'à trois heures avant le temps cédulé de la chirurgie et la médication orale serait prise avec $300 \mathrm{ml}$ d'eau jusqu'à une heure avant la chirurgie.

3 L'administration préopératoire de bloqueurs des récepteurs $\mathrm{H} 2$ soit considérée pour des patients ayant un risque accru de régurgitation et d'aspiration du contenu gastrique.

\section{References}

1 Beaumont $W$. Gastric juice and the physiology of digestion. Plattsburgh: Allen, 1833; 277: 159-60.

2 Snow J. On Chloroform and Other Anaesthetics. London: Churchill, 1858: 74-5.

3 Lister J. On Anaesthetics, Part III. In: Holmes' System of Surgery, Vol III, 3rd ed. London, 1883. (Reprinted in: The Collected Papers of Joseph Lister, Birmingham: The Classics of Medicine Library 1979: 171-2).

4 Boyle HEG, Hewer CL. Practical Anaesthetics, 3rd ed. London: Frowde, Hodder and Stoughton, 1923: 9.

5 Scarr M, Maltby JR, Jani Ket al. Volume and acidity of residual gastric fluid after oral fluid ingestion before elective ambulatory surgery. Can Med Assoc J. 1989; 141: 1151-4.

6 Splinter WM, Stewart JA, Muir JG. Large volumes of apple juice preoperativeiy do not affect gastric $\mathrm{pH}$ and volume in children. Can J Anaesth 1990; 37: 36-9.

7 Sandhar BK, Goresky GV, Maltby JR, Shaffer EA. Effects of oral liquids and ranitidine on gastric fluid volume and $\mathrm{pH}$ in children undergoing outpatient surgery. Anesthesiology $1989 ; 71: 327-30$.

8 Hutchinson A, Maltby JR, Reid CRG. Gastric fluid volume and $\mathrm{pH}$ in elective inpatients. Part $\mathrm{I}$ : coffee or orange juice versus overnight fast. Can J Anaesth 1988; 35: 12-5.
9 Nimmo WS. Drugs, diseases and altered gastric emptying. Clin Pharmacokinet 1976; 1: 189-203.

10 Moore JG, Christian PE, Coleman RE. Gastric emplying of varying meal weight and composition in man. Evaluation by dual liquid and solid phase method. Dig Dis Sci 1981; 26: 16-22.

11 Roberts RB, Shirley MA. Reducing the risk of aspiration during cesarean section. Anesth Analg 1974; 53: 859-68.

12 Raidoo DM, Brock-Utre JG, Rocke DA, Marszalek A, Englebrecht $H E$. The critical volume in acid aspiration. Anesthesiology 1989; 71: A879.

13 Guidelines to the practice of anacsthesia as recommended by the Canadian Anaesthetists' Society. The Canadian Anaesthctists' Society, 187 Gerrard Street East, Toronto, M5A 2E5, 1987. 